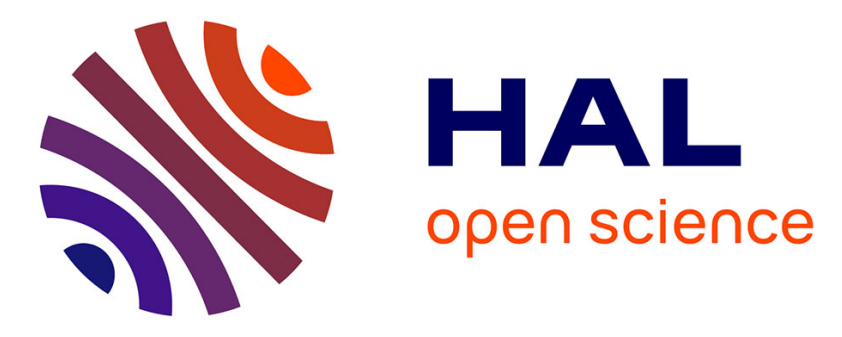

\title{
Spinopelvic sagittal alignment of patients with transfemoral amputation
}

Julia Facione, Coralie Villa, Xavier Bonnet, Cédric Barrey, Marie

Thomas-Pohl, Eric Lapeyre, François Lavaste, Hélène Pillet, Wafa Skalli

\section{To cite this version:}

Julia Facione, Coralie Villa, Xavier Bonnet, Cédric Barrey, Marie Thomas-Pohl, et al.. Spinopelvic sagittal alignment of patients with transfemoral amputation. European Spine Journal, 2019, 28 (9), pp.1920-1928. 10.1007/s00586-019-06017-x . hal-02611123

HAL Id: hal-02611123

https://hal.science/hal-02611123

Submitted on 18 May 2020

HAL is a multi-disciplinary open access archive for the deposit and dissemination of scientific research documents, whether they are published or not. The documents may come from teaching and research institutions in France or abroad, or from public or private research centers.
L'archive ouverte pluridisciplinaire HAL, est destinée au dépôt et à la diffusion de documents scientifiques de niveau recherche, publiés ou non, émanant des établissements d'enseignement et de recherche français ou étrangers, des laboratoires publics ou privés. 


\title{
Spinopelvic sagittal alignment of patients with transfemoral amputation
}

\author{
Julia Facione ${ }^{1,2}$ - Coralie Villa ${ }^{2,3} \cdot$ Xavier Bonnet $^{2} \cdot$ Cédric Barrey $^{4} \cdot$ Marie Thomas-Pohl $^{1,2} \cdot$ Eric Lapeyre $^{1}$. \\ François Lavaste ${ }^{2,3} \cdot$ Hélène Pillet $^{2} \cdot$ Wafa Skalli $^{2}$
}

\begin{abstract}
Purpose This study aims to describe the spinopelvic sagittal alignment in transfemoral amputees (TFAs) from a radiologic study of the spine with a postural approach to better understand the high prevalence of low back pain (LBP) in this population. Methods TFAs underwent X-rays with 3-D reconstructions of the full spine and pelvis. Sagittal parameters were analyzed and compared to the literature. Differences between TFAs with and without LBP were also observed.

Results Twelve subjects have been prospectively included (TFA-LBP group $(n=5)$ and TFA-NoP group $(n=7))$. Four of the five subjects of the TFA-LBP group and two of the seven in TFAs-NoP group had an imbalanced sagittal posture, especially regarding the T9-tilt, significantly higher in the TFA-LBP group than in the TFA-NoP $(p=0.046)$. Eight subjects (6 TFA-NoP and 2 TFA-LBP) had abnormal low value of thoracic kyphosis (TK). Moreover, the mean angle of TK in the TFA-NoP group was lower than in the TFA-LBP group $(p=0.0511)$.

Conclusion In the considered sample, TFAs often present a sagittal imbalance. A low TK angle seems to be associated with the absence of LBP. It can be hypothesized that this compensatory mechanism of the sagittal imbalance is the most accessible in this population. This study emphasizes the importance of considering the sagittal balance of the pelvis and the spine in patients with a TFA to better understand the high prevalence of LBP in this population. It should be completed by the analysis of the spinopelvic balance and the lower limbs in 3D.
\end{abstract}

Keywords Amputation $\cdot$ Low back pain $\cdot$ Posture $\cdot$ Sagittal alignment $\cdot$ Spine

Julia Facione

Julia.9916@hotmail.fr

Extended author information available on the last page of the article

\section{Introduction}

The prevalence of low back pain (LBP) has been shown to be higher in the population of lower-extremity amputees (LEAs) than in the general population, particularly in transfemoral amputees (TFAs) [1,2]. Furthermore, lumbar 
pain impacts the function and quality of life of these patients $[1,3]$. A recent systematic review of the literature highlights that postural issue is one of the five phenomena associated with LBP in LEAs [4]. However, concerning the notions of spinopelvic sagittal alignment and economical posture, there is no study including LEAs to our knowledge. In the general population, it is now accepted that a misalignment could induce an imbalanced or noneconomical posture, causing an increase in the loads on the spinal structures. Subsequently, it can cause a overuse of trunk muscles to compensate the imbalance [5-11]. Thus, changes in the spinal posture can lead to pain due to premature lesions of structures and muscular efforts. The sagittal imbalance and the compensatory mechanisms involved to recover an economical posture are the key factors to understand the spinopelvic alignment [12]. To our knowledge, only one study reports radiographic analysis of the LEAs' spine, but the notion of sagittal balance was not addressed [13].

In this context, it was hypothesized that the spinopelvic sagittal alignment may be altered following a lower-limb amputation. The static disorders could be either a direct consequence of prosthesis-related support changes, or a result of compensatory mechanisms, or both.

The aim of the present study was to assess the spinopelvic sagittal alignment of TFAs to contribute to the understanding of the mechanisms involved in the occurrence of LBP in this population. TFAs were separated into two groups: with and without LBP, and were compared with a population of able-bodied subjects.

\section{Materials and methods}

\section{Subject details}

The sample was composed of prospectively included volun-teers with a unilateral and traumatic transfemoral amputa-tion, able to walk without walking aids and free from mus-culoskeletal disorders on the intact side. To overcome the effect of aging on the spine, subjects older than 60 were not included. Patients wore their own prosthesis for the study and were classified into two groups depending on the answer to the question "Do you suffer from low back pain?": TFA-LBP (TFA and low back pain) and TFA-NoP (TFA and no pain). In the TFALBP group, pain was quantified with a VAS (Visual Analogue Scale) and the impact of LBP on their quality of life evaluated with the ODI (Oswestry Dis-ability Index) [14]. In both groups, sedentarity was evaluated by the patient in number of hours per day spent in a seated position. Finally, the daily duration of prosthesis use was estimated in number of hours per day

\section{Imaging}

To analyze the postural alignment, biplanar low-dose $\mathrm{X}$-rays of the full spine were obtained with the EOSTM system (EOSTM Imaging) [15]. Patients were asked to stand up in the standardized free-standing position, adapted from Faro et al. [16], with the hands-on the cheekbones and with shifted feet positioned as described by Chaibi et al. [17]. Special care was taken to get a natural and comfortable posture.

\section{Data processing}

Using validated reconstruction techniques [18], a 3D patient-specific model was obtained to calculate sagittal parameters [7, 9, 19]; in the anatomo-gravital frame, the frontal plane is the vertical plane going through both acetabulum centers, and transversal and sagittal planes are orthogonal to the frontal plane. The origin of the frame is the center of the bi-coxofemoral segment [20].

\section{Spinal curvatures}

Figure 1a illustrates and defines the spinal curvature parameters computed in this study. Cervical lordosis (CL), thoracic kyphosis (TK) and maximal lumbar lordosis (MLL) were measured according to the method described by Cobb [21]. Lordosis was negative, and kyphosis was positive.

\section{Sagittal pelvic parameters}

Figure $1 \mathrm{~b}$ illustrates and defines the three sagittal pelvic parameters computed in this study: Pelvic incidence (PI), pelvic tilt (PT) and sacral slope (SS). The PI stands for the relative position of the sacral plate with respect to the femoral heads and must be considered as a key parameter of the sagittal analysis $[6,8]$. Indeed, PI is the only morphological parameter determined after the end of growth, fixed for each subject, and is not influenced by position or aging. This angle is the algebraic sum of the pelvic tilt and the sacral slope, the other two pelvic parameters [6]. The normality corridor is considered between $44^{\circ}$ (Mean - 1SD) and $65^{\circ}$ $($ Mean $+1 \mathrm{SD})[7,8]$. The variations of the SS are associated with inversely proportional variations of the PT. Both parameters reflect the sagittal orientation of the pelvis.

\section{Sagittal balance parameters}

Figure 1c-e illustrates and defines the sagittal balance parameters computed in this study: $O D-H A$, sagittal vertical axis (SVA), $T 9$ tilt. 

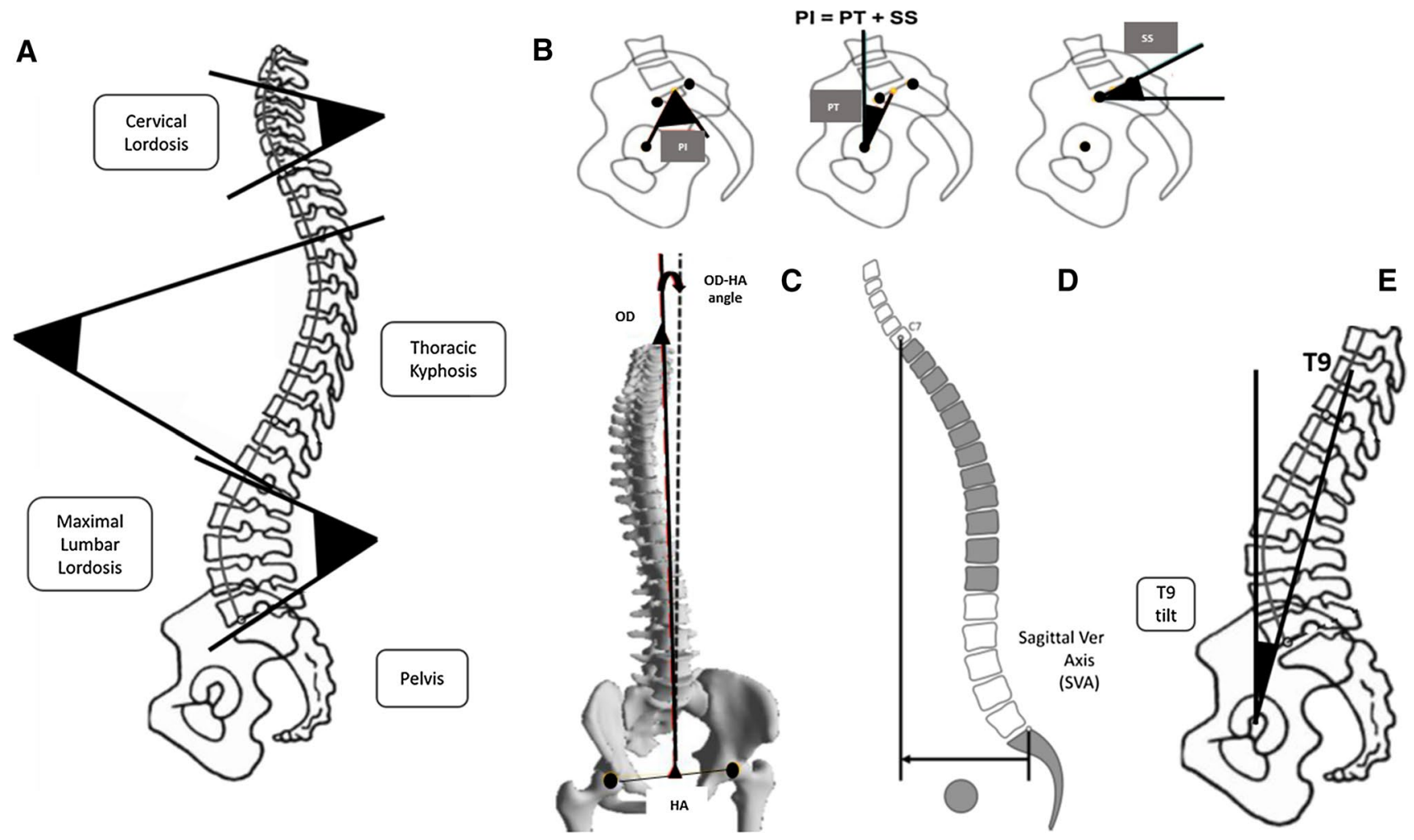

Fig. 1 a Spinal curvatures' measurement, adapted from Amabile's $\mathrm{PhD}$ thesis: Cervical lordosis $(C L)$ : angle between the $\mathrm{C} 3$ upper vertebral plate and C7 lower vertebral plate [20]. Thoracic kyphosis (TK): angle between the T4 upper vertebral plate and T12 lower vertebral plate [8, 32]. Maximal lumbar lordosis (MLL): angle between the superior sacral plate and the most tilted vertebral plate (transitional vertebra between LL and TK) $[7,8]$; b pelvic parameters: PI, adapted from Le Huec et al. [19]: Pelvic incidence (PI): angle between the line connecting the center of the femoral heads and the middle of the sacral plate perpendicular to the sacral plate. Pelvic tilt (PT): angle between the line connecting the center of the femoral heads and the middle of the sacral plate and the vertical. Sacral slope (SS): angle between the sacral plate line and the horizontal $[11,13]$; c OD-HA, adapted from Amabile et al. [20]: angle between the line joining the most superior point of dentiform apophyse of $\mathrm{C} 2$

\section{Parameters analysis}

For each parameter, the normality corridors of a reference population were defined from previous studies in able-bodied subjects carried out in the same laboratory. As proposed in the work of Amabile et al., the age of the subjects was taken into account for the OD-HA, the SVA and the cervical lordosis. Subjects were considered young subjects if $<49$ years old [20] or older subjects if $\geq 49$ years old [22]. For all other parameters, normality corridors were independent on age [8]. Normal values were within [Mean $\pm 1 \mathrm{SD}]$, Subnormal High were between (Mean + 1SD) and (Mean + 2SD), Subnormal Low between (Mean - 1SD) and (Mean - 2SD), and Abnormal values were out of $[$ Mean $\pm 2 S D]$. Parameters were all
(OD) and the middle of acetabulum centers (HA) with the vertical, which is an invariant parameter in the young and in the elderly general populations $[20,22]$. In a balanced posture, the head is over the pelvis and the OD-HA is close to zero; $\mathbf{d}$ Sagittal vertical axis: SVA, adapted from Diebo et al. [32]: posteroanterior distance between the C7 plumb line and the posterosuperior point of the sacral plate. SVA describes the anteroposterior balance. This is the most commonly used parameter to characterize the sagittal balance. In a balanced posture, the cervical spine is over the pelvis and the SVA is close to zero [12, 32]; e T9-tilt, adapted from Amabile's PhD thesis: angle between the line connecting the vertebral body center of T9 to HA and the vertical. This angle can be considered as an indicator of the position of the trunk center of gravity (including head and arm). In a balanced posture, T9 projects behind the femoral heads [9]

individually analyzed for each subject. The sagittal balance (expected values of PT and LL [8]) was analyzed to estimate the economical character of the posture of each subject. Parameters involved in the compensatory mechanisms were: CL (cervical hyperlordosis), TK (decrease in the TK) and PT (pelvis backtilt). Then, differences between the two groups were observed.

\section{Ethics}

Following the protocol approved by the Ethics Committee (Comité de Protection des Personnes, CPP NX06036), biplanar X-ray radiographs were obtained after written informed consent. 


\section{Statistical analysis}

To find the differences between the two groups, a Student's $t$ test was used, considering the normality of the distribution as already shown in previous studies for all pelvic and spinal parameters $[8,9,20]$. Concerning the anthropometrical parameters, variances were compared with a Fisher test.

\section{Results}

\section{Anthropometrical parameters}

Twelve subjects (10 males and two females) corresponding to the inclusion criteria have been included between February and May 2017. The variances were homogeneous, allowing to use the Student's $t$ test. The two groups (TFA-LBP $n=5$ and TFA-NoP $n=7$ ) were not significantly different in terms of gender, age, body mass index (BMI), time since amputation, duration of prosthesis use per day and sedentarity. Eleven subjects were equipped with a microprocessorcontrolled knee, and one subject had a mechanical knee. In the TFA-LBP group, the mean minimal Visual Analogue Scale (VAS) pain was 2.1/10 (SD 1.1) and the mean maximal VAS pain was $6.5 / 10$ (SD 1.4). The impact of LBP in the TFA-LBP group was estimated on average to $16.4 \%$ with the ODI. The anthropometrical parameters of the sample are given in Table 1.

\section{Sagittal parameters}

The sagittal parameters of the sample are given in Table 2 . To illustrate, the radiographs of two TFA-LBP subjects are presented in Fig. 2.

Spinal curvatures: Among the twelve TFAs, CL values were highly variable (Fig. 3, top). All the CL values were in the normal corridor except for two subjects (one in each group).
CL was negative (pronounced lordosis) in four of the five TFA-LBP subjects and two of the seven TFA-NoP subjects. Concerning TK, eight subjects (six NoP and two LBP) had abnormal low values of TK (Fig. 3, bottom). Moreover, the mean TK was lower in TFA-NoP subjects than in TFAsLBP, $p=0.0511$ (Fig. 4). Concerning MLL, there was no difference in the mean angle between the two groups. For each subject, the predicted MLL expected in an economical posture inferred from SS was compared to the real measured MLL and is shown in Fig. 4 (top). In two subjects of the TFA-LBP group, an increase in the MLL compared to the expected value was observed. On the contrary, for all TFA-NoP subjects, the MLL was suitable and often below the expected values.

Pelvic parameters: According to the reference population [8], all PIs were normal or subnormal except for one subject $\left(30.5^{\circ}\right)$ in the TFA-LBP group. Compared to the predicted values expected in an economical posture, anterior PT was observed in two TFA-NoP subjects and two TFA-LBP subjects and posterior PT was observed in one subject of each group (Fig. 4, bottom).

Sagittal balance parameters: OD-HA was normal in six out of seven individuals in the TFA-NoP group and one out of five in the TFA-LBP group. The SVA was abnormal in one subject of each group. The T9-tilt was normal in two out of five subjects of the TFA-LBP group, while T9-tilt was always normal in the TFA-NoP group; difference is statistically demonstrated, $p=0.046$ (Fig. 5).

\section{Discussion}

\section{Strengths and limitations of the study}

To our knowledge, this study is the first to describe the spinopelvic sagittal alignment in a population of lowerlimb amputees [23-27]. The statistical analysis was limited
Table 1 Anthropometrical parameters of the sample

\begin{tabular}{lllll}
\hline Subject details & $\begin{array}{l}\text { Total sample }(n=12) \\
\text { mean/range/SD }\end{array}$ & $\begin{array}{l}\text { TFA-LBP }(n=5) \\
\text { mean/range/SD }\end{array}$ & $\begin{array}{l}\text { TFA-NoP }(n=7) \\
\text { mean/range/SD }\end{array}$ & $\begin{array}{l}p \text { value, } \\
\text { LBP vs } \\
\text { NoP }\end{array}$ \\
\hline Male/female & $2 \mathrm{~F} / 10 \mathrm{M}$ & $1 \mathrm{~F} / 4 \mathrm{M}$ & $1 \mathrm{~F} / 6 \mathrm{M}$ & NA \\
Age (y) & $39 / 24-58 / 11.8$ & $43 / 26-58 / 13.7$ & $37 / 24-49 / 10.6$ & 0.454 \\
BMI (Kg/m2) & $22.9 / 17-29 / 4.2$ & $25.5 / 21.9-29.3 / 2.8$ & $21.0 / 16.6-28.3 / 4.1$ & 0.058 \\
Time since TFA (y) & $10 / 1-31 / 10$ & $10 / 2.5-31 / 11.9$ & $11 / 0.7-25 / 9.7$ & 0.921 \\
Sedentarity (h/d) & $6.5 / 3-9 / 2$ & $7.6 / 6-9 / 1.1$ & $5.5 / 3-8 / 2.1$ & 0.075 \\
Prosthesis use (h/d) & $13 / 7.5-16 / 2.7$ & $13 / 10-16 / 2.4$ & $12.6 / 7.5-15 / 2.9$ & 0.795 \\
\hline
\end{tabular}

$B M I$ body mass index, $F$ female, $L B P$ low back pain, $M$ male, $N o P$ no pain, $S D$ standard deviation, $T F A$ transfemoral amputation, $N A$ not applicable 


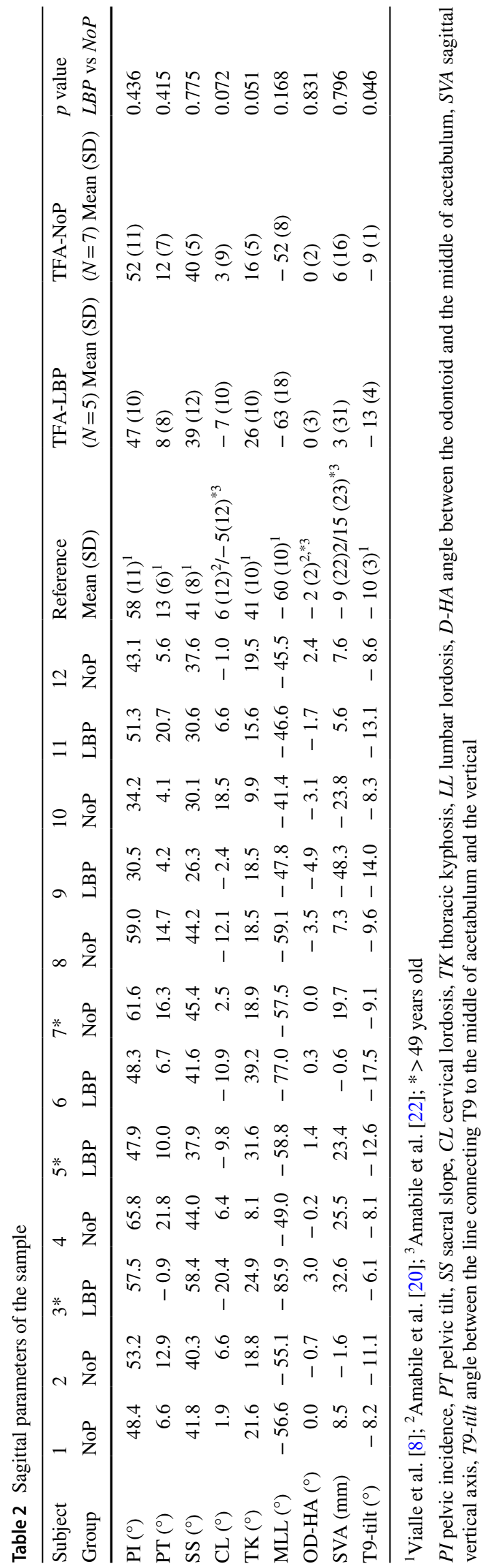

because of the number of subjects. It must also be noticed that the present study only focused on the spinal and pelvic parameters excluding the analysis of lower-limb parameters. The variability of the results was another limitation. However, as emphasized by Highsmith et al., the understanding of LBP in LEAs remains a knowledge gap, with too few experimental studies [4]. The present contribution brings original elements in the understanding of posture in this population.

\section{Prevalence of LBP}

The prevalence of LBP estimated to $41.7 \%$ in our sample was lower than the prevalence previously reported in the literature for TFAs [1, 3, 28, 29]. However, Highsmith et al. reported a prevalence of LBP in LEAs from $36 \%$ to $89 \%$ in the recent and systematic review of the literature. With a prevalence of $41.7 \%$, our sample showed a higher prevalence of LBP than in the non-amputee general population (estimated from 15 to $25 \%$ ). Moreover, the subjects included in the present study reported a low impact of the LBP on their daily life compared to previous observations $[1,3]$. This discrepancy could be explained by the age of the subjects included in our sample to avoid confounding the effects of amputation with the effects of aging on the spine. However, considering a recent study about the cutoff value of the ODI, three of the five subjects of the TFA-LBP group reported an ODI score higher than the cutoff value (12.0) [30].

\section{Sagittal balance}

First, we showed that sagittal balance parameters were often in the subnormal or abnormal corridors, especially regarding the OD-HA and T9-tilt parameters and noticeably more in the TFAs-LBP group. These data suggest that TFAs may be more often in an unbalanced posture than non-amputees. Thus, head offset relative to the pelvis (quantified through OD-HA parameter) was forward in three cases and behind in one case in the TFA-LBP group although it is considered as an invariant parameter in the non-amputee population even in the older one, known to frequently present anterior imbalance [22]. One explanation would be attributed to the prosthetic alignment, which usually includes the positioning of the load line in the middle of the prosthetic foot to facilitate the gait [31]. It is known that in the able-bodied population, subjects with sagittal (anterior) imbalance used compensatory mechanisms to regain a new sagittal balance: increase in the cervical lordosis, decrease in the thoracic kyphosis, decrease in the pelvic tilt, flexion of the knees and extension of the ankles [12]. It might be suspected that TFA-NoP subjects were able to regain a correct sagittal balance, while TFA-LBP subjects were not. Indeed, a significant anteriorly shifted position of T9 in TFA-NoP compared to TFA-LBP 
Fig. 2 Lateral radiograph of the full spine in two TFA-LBP subjects (subnormal values have one arrow; abnormal values have two arrows and normal values have a "N"). ${ }^{1}$ Normality corridor of pelvic and spinal parameters (Vialle et al. [8]); ${ }^{2}$ Relation with PI from Vialle equation (Vialle et al. [8]); ${ }^{3}$ Normality corridor of OD-HA (Amabile et al. [20, 22])
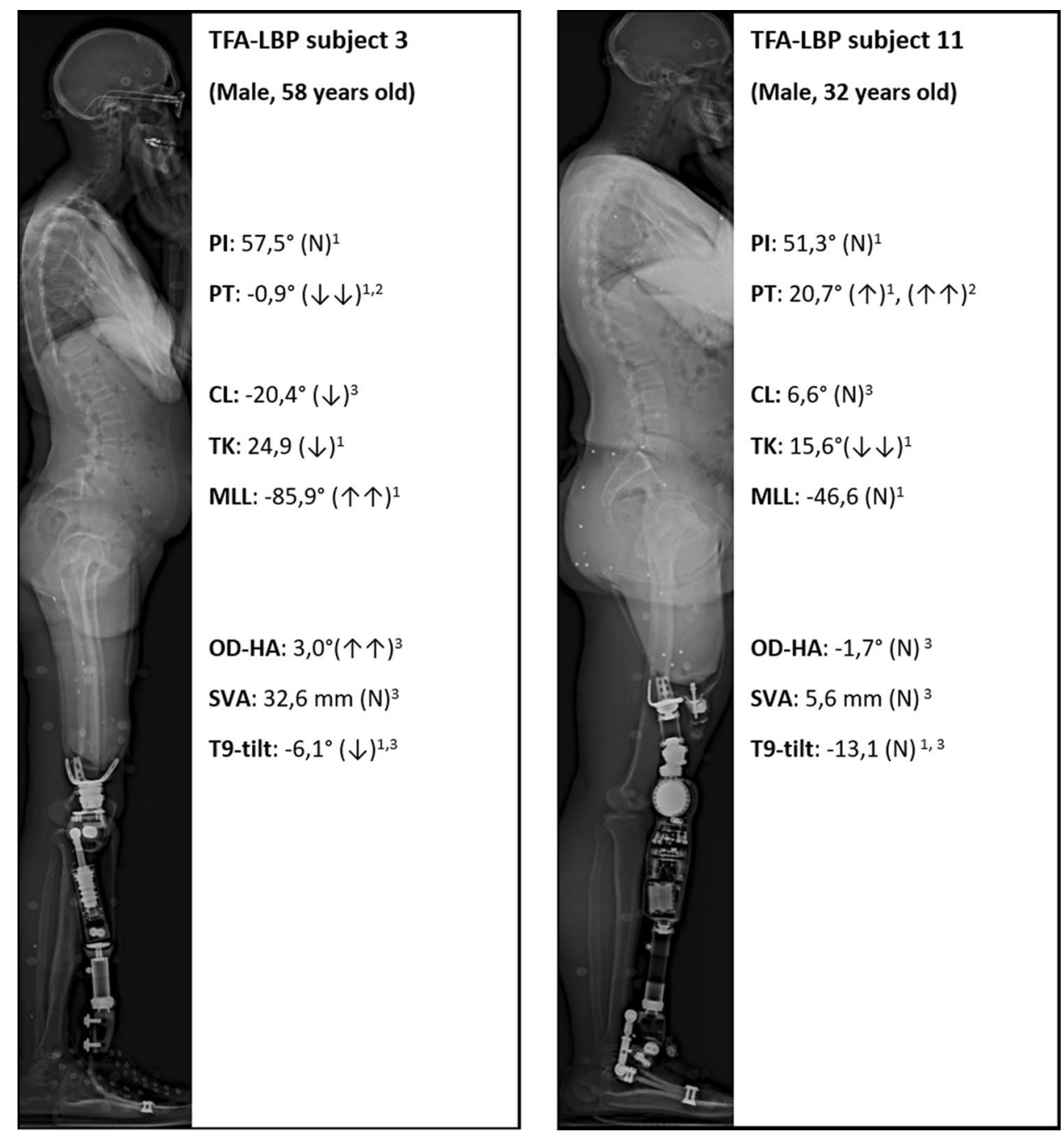

$(p<0.05)$ was noticed raising the idea of a possible compensatory mechanism at the thoracic level. Thus, OD-HA and T9-tilt could be interesting balance parameters in the TFA population to assess the sagittal imbalance. On the contrary, even if currently used in clinical practice, SVA is proved to be less relevant in our sample.

\section{Spinal parameters}

Focusing on the cervical area, although included in normal or subnormal values, the cervical lordosis (CL) seems to be more pronounced in the TFA-LBP group than in the TFANoP. The increase in the CL, observed in the TFA-LBP group, is a typical compensatory mechanism to maintain the horizontality of the gaze in case of anterior imbalance [12].

The TK was the most frequently abnormal parameter (often decreased) in the entire sample when compared to non-amputee (abnormal low values in 8 subjects including
6 TFA-NoP). By limiting the forward-shifting of the trunk, the decrease in TK is one of the well-known compensatory mechanisms of anterior imbalance, especially in young subjects with flexible spine [12]. TK was very low for most of the correctly balanced subjects suggesting its decrease as an effective way to compensate sagittal imbalance.

As regards the MLL, the values of TFA-LBP subjects were globally close to the expected values inferred from the SS values in an economical balance, except in two subjects demonstrating an increased MLL (associated with anterior PT). On the contrary, the MLL values of TFA-NoP were always equivalent or lower than the expected values.

\section{Pelvic parameters}

The PT of TFA subjects was close to the expected value predicted from the PI value in an economical posture for 

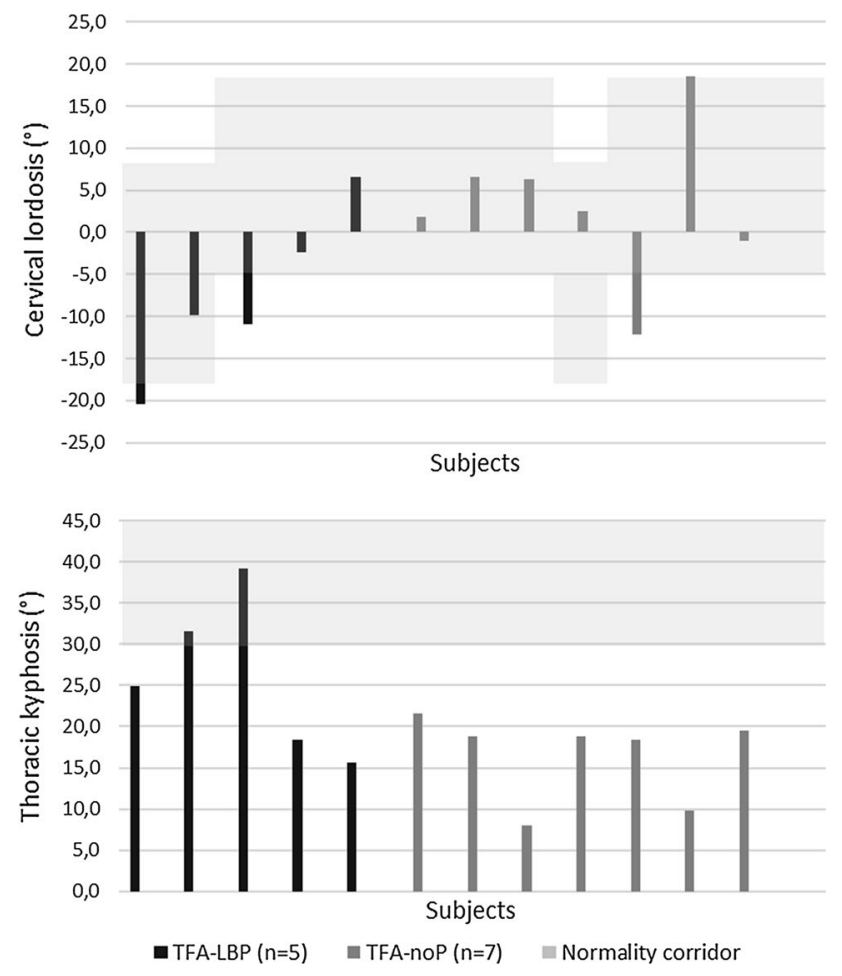

Fig. 3 Top: Details of cervical lordosis values in the sample with normality corridors from the reference population of Amabile et al. [20, 22] depending on the subjects' age: CL TFA-LBP vs CL TFA-NoP, $p=0.0876$. Bottom: Details of thoracic kyphosis values in the sample, with normality corridors from the reference population of Vialle et al. [8]: TK TFA-LBP vs TK TFA-NoP, $p=0.0511$

six of the twelve subjects (four out of seven in the TFANoP group and two out of five in the TFA-LBP group). PT was decreased (anterior PT) in four subjects (two in each group) and increased (posterior PT) in two subjects (one in each group). The variability of the results prevents a deeper analysis.

\section{Reflexions about compensatory mechanisms}

Among all the compensatory mechanisms of the sagittal imbalance [12, 19, 32], in the present study on TFAs, either a reduction in the thoracic curve magnitude and at least an increase in the cervical lordosis was found. The thoracic compensation was associated with the absence of pain, suggesting its effectiveness, while the cervical compensation was rather associated with the presence of pain. As regards pelvis compensatory mechanisms, the increase in PT was rarely observed, while this mechanism is very common in the sagittal imbalanced older subjects. We suggest that the posterior part of the femoral socket, in particular with an "ischial containment socket," could limit the increase in PT (Fig. 2, subject 3). Finally, with a prosthetic leg, knee flexion and ankle extension cannot be used to compensate the sagittal imbalance.

\section{Perspectives}

Even if the notion of equilibrium has been essentially considered in the sagittal plane, coronal imbalance should also be observed in TFAs because lower-limb length discrepancy is common in this population. A complete 3-D approach of the spinal posture disorders potentially involved in the occurrence of LBP in the TFAs would be useful for clinical practice. Moreover, it could also be relevant to assess the parameters of the lower limbs, for example the sacro-femoral angle and the pelvic shift as reported by some authors in patients with spinal deformity (role of pelvic translation and lower-extremity compensation to maintain gravity line position in spinal deformity) [33].

\section{Conclusion}

This study assessed the sagittal alignment of a population of TFAs. Altered sagittal balance parameters were found in some subjects, maybe more frequently in TFAs with LBP than in TFAs without LBP, which is consistent with the hypothesis that imbalanced posture is associated with LBP. Compensatory mechanisms of the sagittal imbalance known in the non-amputee population may be less effective in TFAs because they also involve the pelvis and the lower limbs. This could explain the frequency of imbalanced posture in the TFAs. Only the decrease in the thoracic kyphosis seems to be associated with the absence of back pain, suggesting that this mechanism could be an effective and accessible way to maintain sagittal balance.

Finally, the main perspective of this work is to study not only the sagittal plane but also the coronal and horizontal 
Fig. 4 Adaptation of PT and MLL (correlation with other parameters). Calculated $\mathrm{LL}=-16-1.06 * \mathrm{SS}$; Calculated $\mathrm{PT}=-7+0.37 * \mathrm{PI} \pm 1.5$ (Vialle et al. [8])

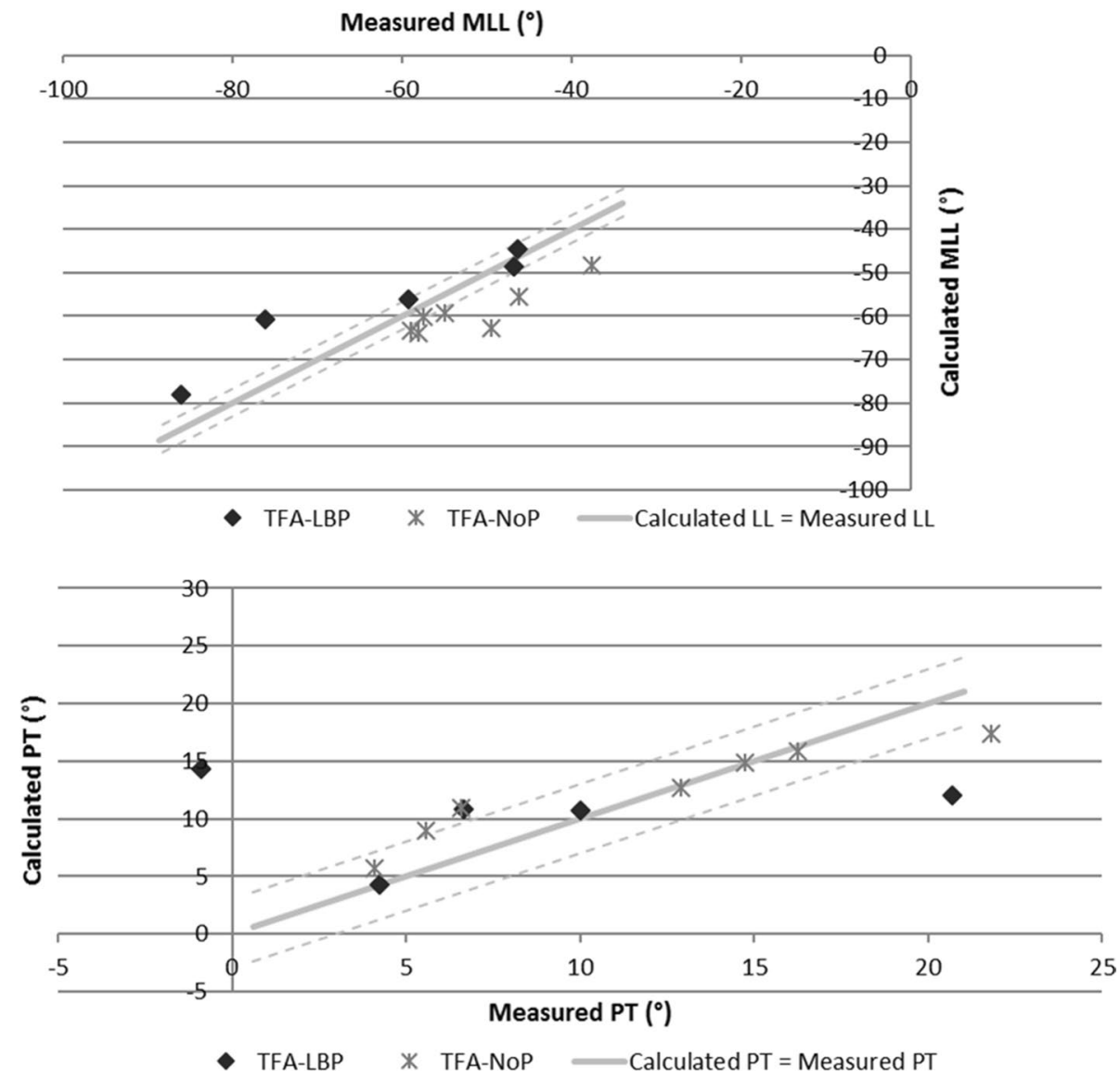

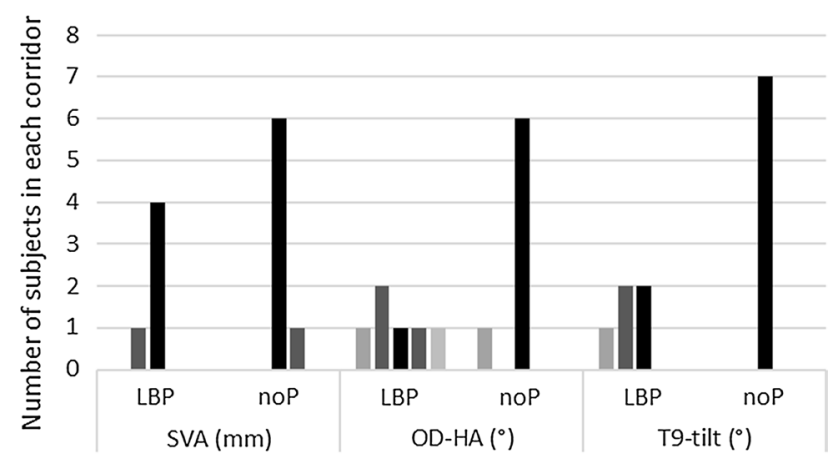

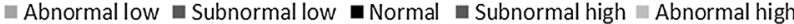

Fig. 5 Normality corridors of sagittal balance parameters in the sample $(N=12)$. OD-HA, angle between the odontoid and the middle of acetabulum; SVA, sagittal vertical axis; T9-tilt, angle between the line connecting T9 to the middle of acetabulum and the vertical. Normal values were within [Mean $\pm 1 \mathrm{SD}]$, Subnormal High were between $($ Mean $+1 \mathrm{SD})$ and $($ Mean $+2 \mathrm{SD})$, Subnormal Low between (Mean - 1SD) and (Mean-2SD), and Abnormal values were out of [Mean $\pm 2 S D$ ], from Amabile et al. [20, 22]

planes in order to better understand the high prevalence of LBP in the TFAs and give the key to clinicians to manage LBP in amputee patients.

\section{Compliance with ethical standards}

Conflict of interest The authors declare that they have no conflict of interest.

\section{References}

1. Ehde DM, Smith DG, Czerniecki JM, Campbell KM, Malchow DM, Robinson LR (2001) Back pain as a secondary disability in persons with lower limb amputations. Arch Phys Med Rehabil 82(6):731-734

2. Gailey R, Allen K, Castles J, Kucharik J, Roeder M (2008) Review of secondary physical conditions associated with lowerlimb amputation and long-term prosthesis use. J Rehabil Res Dev 45(1):15-29

3. Smith DG, Ehde DM, Legro MW, Reiber GE, del Aguila MBD (1999) Phantom limb, residual limb, and back pain after lower extremity amputations. Clin Orthop Relat Res 361:29-38

4. Highsmith MJ, Goff LM, Lewandowski AL, Farrokhi S, Hendershot BD, Hill OT et al (2018) Low back pain in persons with lower extremity amputation: a systematic review of the literature. Spine J 19:552-563

5. Legaye J, Hecquet J, Marty C et al (1993) Sagital equilibration of the Spine: relationship between pelvis and sagittal spinal curves in the standing position. Rachis 5(5):215-226 
6. Legaye J, Duval-Beaupère G, Hecquet J, Marty C (1998) Pelvic incidence: a fundamental pelvic parameter for three-dimensional regulation of spinal sagittal curves. Eur Spine J 7:99-103

7. Legaye J, Duval-Beaupère G (2005) Sagittal plane alignment of the spine and gravity a radiological and clinical evaluation. Acta Orthop Belg 71(2):213-220

8. Vialle R, Levassor N, Rilladon L, Templier A, Skalli W, Guigui P (2005) Radiographic analysis of the sagittal alignment and balance of the spine in asymtomatic subjects. J Bone Jt Surg Am. 87(2):260-267

9. Boulay C, Tardieu C, Hecquet J, Benaim C, Mouilleseaux B, Marty C et al (2006) Sagittal alignment of spine and pelvis regulated by pelvic incidence: standard values and prediction of lordosis. Eur Spine J 15(4):415-422

10. Galbusera F, Wilke HJ, Brayda-Bruno M, Costa F, Fornari M (2013) Influence of sagittal balance on spinal lumbar loads: a numerical approach. Clin Biomech 28(4):370-377

11. Amabile C, Choisne J, Nérot A, Pillet H, Skalli W (2016) Determination of a new uniform thorax density representative of the living population from $3 \mathrm{D}$ external body shape modeling. J Biomech 49(7):1162-1169

12. Barrey C, Roussouly P, Le Huec JC, D’Acunzi G, Perrin G (2013) Compensatory mechanisms contributing to keep the sagittal balance of the spine. Eur Spine J 22(6):834-841

13. Mj B, Roman V, Wright V (1978) Bone and joint changes in lower limb amputees. Ann Rheum Dis 37(3):252-254

14. Vogler D, Paillex R, Norberg M, de Goumoëns P, Cabri J (2008) Validation transculturelle de l'Oswestry disability index en français. Ann Réadaptation Médecine Phys 51(5):379-385

15. Dubousset J, Charpak G, Skalli W, Deguise J, Kalifa G (2010) EOS: a new imaging system with low dose radiation in standing position for spine and bone and joint disorders. J Musculoskelet Res 13(01):1-12

16. Faro FD, Marks MC, Pawelek J, Newton P (2004) Evaluation of a functional position for lateral radiograph acquisition in adolescent idiopathic scoliosis. Spine 29(20):2284-2289

17. Chaibi Y, Cresson T, Aubert B, Hausselle J, Neyret P, Hauger O et al (2012) Fast 3D reconstruction of the lower limb using a parametric model and statistical inferences and clinical measurements calculation from biplanar X-rays. Comput Methods Biomech Biomed Eng 15(5):457-466

18. Humbert L, De Guise JA, Aubert B, Godbout B, Skalli W (2009) $3 \mathrm{D}$ reconstruction of the spine from biplanar X-rays using parametric models based on transversal and longitudinal inferences. Med Eng Phys 31:681-687

19. Le Huec JC, Saddiki R, Franke J, Rigal J, Aunoble S (2011) Equilibrium of the human body and the gravity line: the basics. Eur Spine J 20(S5):558-563

20. Amabile C, Pillet H, Lafage V, Barrey C, Vital J-M, Skalli W (2016) A new quasi-invariant parameter characterizing the postural alignment of young asymptomatic adults. Eur Spine J 25(11):3666-3674

21. Cobb J. Outline for the study of scoliosis, Instr. Course Lect. In: Am Acad Orthop Surg. Ann Arbor, Michigan; 1948. p. 261

22. Amabile C, Le Huec J-C, Skalli W (2018) Invariance of headpelvis alignment and compensatory mechanisms for asymptomatic adults older than 49 years. Eur Spine J 27(2):458-466

23. Morgenroth DC, Shakir A, Orendurff MS, Czerniecki JM (2009) Low-back pain in transfemoral amputees is there a correlation with static or dynamic leg-length discrepancy? Am J Phys Med Rehabil 88(2):108-113

24. Morgenroth DC, Orendurff MS, Shakir A, Segal A, Shofer J, Czerniecki JM (2010) The relationship between lumbar spine kinematics during gait and low-back pain in transfemoral amputees. Am J Phys Med Rehabil 89(8):635-643

25. Hendershot BD, Bazrgari B, Nussbaum MA (2013) Persons with unilateral lower-limb amputation have altered and asymmetric trunk mechanical and neuromuscular behaviors estimated using multidirectional trunk perturbations. J Biomech 46(11):1907-1912

26. Hendershot BD, Nussbaum MA (2013) Persons with lower-limb amputation have impaired trunk postural control while maintaining seated balance. Gait Posture. 38(3):438-442

27. Yoder AJ, Petrella AJ, Silverman AK (2015) Trunk-pelvis motion, joint loads, and muscle forces during walking with a transtibial amputation. Gait Posture 41(3):757-762

28. Ephraim PL, Wegener ST, MacKenzie EJ, Dillingham TRPL (2005) Phantom pain, residual limb pain, and back pain in amputees: results of a national survey. Arch Phys Med Rehabil 86(10):1910-1919

29. Kulkarni J, Gaine W, Buckley J, Rankine J, Adams J (2005) Chronic low back pain in traumatic lower limb amputees. Clin Rehabil 19(1):81-86

30. Tonosu J, Takeshita K, Hara N, Matsudaira K, Kato S, Masuda $\mathrm{K}$ et al (2012) The normative score and the cut-off value of the Oswestry Disability Index (ODI). Eur Spine J 21(8):1596-1602

31. Blumentritt S (1997) A new biomechanical method for determination of static prosthetic alignment. Prosthet Orthot Int 21:107-113

32. Diebo BG, Varghese JJ, Lafage R, Schwab FJ, Lafage V (2015) Sagittal alignment of the spine: What do you need to know? Clin Neurol Neurosurg 2015(139):295-301

33. Ferrero E, Liabaud B, Challier V, Lafage R, Diebo BG, Vira S et al (2016) Role of pelvic translation and lower-extremity compensation to maintain gravity line position in spinal deformity deformity. J Neurosurg Spine 24(March):436-446

\section{Affiliations}

\section{Julia Facione ${ }^{1,2}\left(\right.$ Coralie Villa $^{2,3} \cdot$ Xavier Bonnet $^{2} \cdot$ Cédric Barrey $^{4} \cdot$ Marie Thomas-Pohl $^{1,2} \cdot$ Eric Lapeyre $^{1}$. François Lavaste ${ }^{2,3} \cdot$ Hélène Pillet $^{2} \cdot$ Wafa Skalli $^{2}$}

1 Physical Medicine and Rehabilitation Unit, Hôpital d'Instruction des Armées Percy, 101 avenue Henri, Barbusse, 92140 Clamart, France

2 Arts et Metiers ParisTech, Institut de Biomécanique Humaine Georges Charpak, 151 bd de l'Hopital, 75013 Paris, France
3 INI, Centre d'Etude et de Recherche sur l'Appareillage des Handicapés, BP 50719, 57147 Woippy Cédex, France

4 Department of Neurosurgery and Spine Surgery, P. Wertheimer Hospital, Hospices civils de Lyon, Université Claude Bernard Lyon 1, Lyon, France 